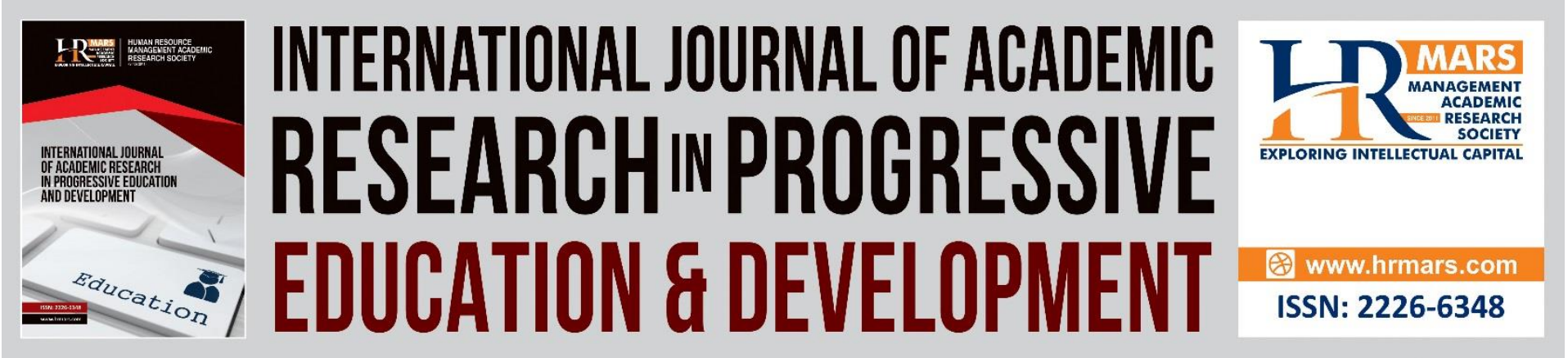

\title{
Analyzing the Insight of Students' Needs for The Development of an Arabic Rhetoric Application Through MOOC
}

\author{
Mohamad Nor Amin Samsun Baharun, Janudin Sardi, Goh Chin Shuang
}

To Link this Article: http://dx.doi.org/10.6007/IJARPED/v10-i3/10706 DOI:10.6007/IJARPED/v10-i3/10706

Received: 13 June 2021, Revised: 16 July 2021, Accepted: 02 August 2021

Published Online: 28 August 2021

In-Text Citation: (Baharun et al., 2021)

To Cite this Article: Baharun, M. N. A. S., Sardi, J., \& Shuang, G. C. (2021). Analyzing the Insight of Students' Needs for The Development of an Arabic Rhetoric Application Through MOOC. International Journal of Academic Research in Progressive Education and Development, 10(3), 320-336.

Copyright: (C) 2021 The Author(s)

Published by Human Resource Management Academic Research Society (www.hrmars.com) This article is published under the Creative Commons Attribution (CC BY 4.0) license. Anyone may reproduce, distribute, translate and create derivative works of this article (for both commercial and non-commercial purposes), subject to full attribution to the original publication and authors. The full terms of this license may be seen at: http://creativecommons.org/licences/by/4.0/legalcode

\section{Vol. 10(3) 2021, Pg. 320 - 336}

\section{http://hrmars.com/index.php/pages/detail/IJARPED}

Full Terms \& Conditions of access and use can be found at http://hrmars.com/index.php/pages/detail/publication-ethics 


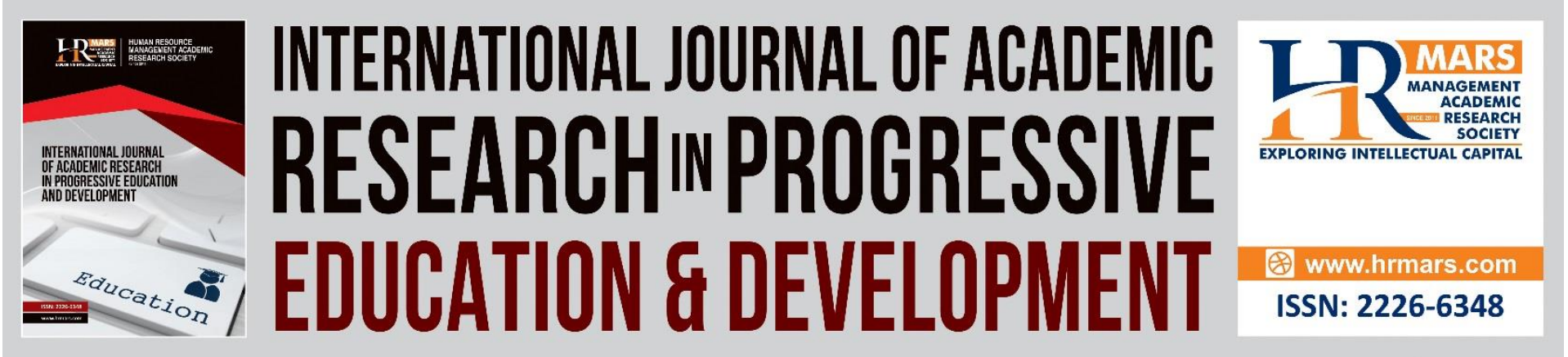

\title{
Analyzing the Insight of Students' Needs for The Development of an Arabic Rhetoric Application Through MOOC
}

\author{
Mohamad Nor Amin Samsun Baharun, Janudin Sardi, Goh \\ Chin Shuang \\ Akademi Pengajian Bahasa, Universiti Teknologi MARA, 40450 Shah Alam, Selangor, \\ Malaysia \\ Email:amin277875@gmail.com, janudin@gmail.com,cassgoh@gmail.com
}

\begin{abstract}
Rhetoric is one of the beautiful arts of expression in Arabic. Unfortunately, many students who learn Arabic Rhetoric are unable to acquire it well due to the lack of interesting materials. Past studies revealed that the mastery level of Arabic Rhetoric among secondary school students is at a moderate level. Therefore, the development of an Arabic Rhetoric application through Massive Open Online Courses (MOOC) should be implemented in facilitating students to understand the concept of Arabic Rhetoric more effectively. Factors such as elaboration of contents via graphics, animation, audios, videos, and texts followed by the provision of exercises may attract students to learn independently as well as to improve their performance. This quantitative study aims to analyze the need for an Arabic Rhetorical application. The respondents comprised 40 form four students enrolled in a secondary school in Shah Alam, Selangor, Malaysia. A questionnaire with a reliability value of 0.931 was administered online via Google Form. It consisted of four sections: i) demographics, ii) Arabic Rhetoric learning approaches, iii) Arabic Rhetoric learning issues, and iv) innovation design in Arabic Rhetoric learning. The analysis of data was conducted via descriptive statistics through percentage, mean, and standard deviation. The findings show that students need innovative design in learning Arabic Rhetoric through an Arabic Rhetorical application in order to facilitate the comprehension of information.
\end{abstract}

Keyword: Arabic Rhetoric, Mastery Level, Massive Open Online Courses (MOOC), Arabic Rhetoric Application, Innovative Design

\section{Introduction}

The subject of Al-Adab Wa Al-Balaghah (Literature and Arabic Rhetoric) was first introduced in 2017 at all Sekolah Agama Bantuan Kerajaan (SABK) (Government Aided Religious Schools) under the supervision of the Ministry of Education Malaysia. Whereas balaghah itself was taught to form four and form five students as a component of the Arabic Language subject since it was introduced in the 90s in most secondary schools throughout Malaysia. Al-Adab 
Wa Al-Balaghah is a subject learned by form four students who enroll in the Arabic language stream and follow the new religious curriculum, namely the Kurikulum Bersepadu Dini dan Tahfiz (KBDT) (Religious and Tahfiz Integrated Curriculum). The curriculum was introduced in 2017 (Dokumen Standard Kurikulum, 2016) and students will continue their studies up to form five to sit for the Sijil Pelajaran Malaysia (SPM) (equivalent to A-level) as well as other subjects like Math, Science, and English.

The new KBDT curriculum has combined the subject of Arabic Rhetoric with Al-Adab (Literature) in one textbook. Students are exposed to Arab Literature in the first part of the textbook and Arabic Rhetoric in the second part (Al-Adab Wa Al-Balaghah Textbook, 2017). Additionally, the teaching of Al-Adab Wa Al-Balaghah is divided into two, in which the theoretical component is taught during form four while the practical implementation of Arabic Rhetoric taught during form four is done in form five. Whereas students who do not follow the KBDT curriculum at religious schools have the option to learn Arabic Rhetoric at secondary schools through 'Arus Perdana' (mainstream curriculum) in the Arabic Language subject in form four and five, and the content of each curriculum is more or less the same. The Arabic Rhetoric application in this study refers to a course application that adopts the Massive Open Online Courses (MOOC) platform, which is free and can be accessed anytime and anywhere.

\section{MOOC}

Massive Open Online Courses (MOOC) is an online learning platform that is attended by many students around the world, which most of the courses are free (Wei et al., 2021). There are various platforms or providers offering the e-learning concept, such as Coursera, EdX, Udacity, Open Learning, and Open Education (Korableva et al., 2019; Chris Piech et al., 2013). Upon registration, students are free to enroll into any courses available. The learning component in these courses include activities such as following teaching videos, answering quizzes, doing assignments, participating in forum activities, and online discussion (Gamage et al., 2020). Teaching videos are the most important element in MOOC. There are many forms of teaching videos delivery that can be created, such as voice over slides design, animation, writing over videos, presence in full screen, and voice over videos plus presence in full screen (Rahim \& Shamsudin, 2019). In short, teaching videos are considered as the main element in the courses that facilitate students' understanding towards the lesson.

Several factors should be considered by MOOC developers to ensure the effectiveness of learning. Learning attitude and learning design influence students' performance significantly in their learning because students' learning behavior determines their ability to continue their learning (Mohamad et al., 2020). Difference in learning styles also affects students' success. Therefore, curriculum planning and implementation of a particular course may affect students' motivation (Qarabesh, 2020). Independent verbal type of cognitive style is closely related to learning effectiveness. From the aspect of teaching, teachers or developers need to consider appropriate teaching materials to improve students' focus when designing courses in MOOC (Chang et al., 2019). Thus, MOOC course developers should consider these elements to ensure that students are motivated to continue their learning.

Furthermore, assessment is another element that requires significant attention to ensure that learning objectives are achieved in MOOC (Wei et al., 2021). The form of assessment in a MOOC course should comprise various levels of difficulty to suit students' level of motivation. Therefore, the development of e-assessment module will strengthen students' 
understanding. Among the exercises that can be executed include multiple-choice questions, crossword puzzles, fill in the blank exercises, true or false questions, and matching questions (Kamarudin, 2020).

\section{Problem Statement}

Discussion on the issue of Arabic Rhetoric mastery among school students has been a pertinent since the 90s. Past literature has reported that students generally face difficulties in mastering the subject (Zaki et al., 2019; Ibrahim et al., 2019; Hasan, 2014; Samah, 2006) particularly on topics such as haqiqah and majaz, majaz mursal, and isti'arah (Ibrahim et al., 2019). In addition, students in public universities have also recorded medium to low mastery level towards Arabic Rhetoric (Hanim et al., 2014).

In response to the issue, teaching techniques and activities are found to be the most significant weaknesses to the issue (Ariffin, 1999). The teaching and learning of Arabic Rhetoric should not focus solely on memorizing definitions (Hakim \& Mustafa, 2016). Furthermore, the learning strategies solely focused on the memorization of examples and concepts, and emphasized less on the application element (Rosni Samah, 2006). Abdul Hakim Abdullah (2008) reported that most students who learned Arabic Rhetoric only focused on methods rather that application in speaking and writing. Subsequently, it placed a limitation on their understanding to the information delivered by the teacher (Sopian et al., 2013; Muhammad et al., 2006). This suggests that the teaching and learning of Arabic Rhetoric should focus on the application element rather than emphasizing solely on memorizing.

Furthermore, teaching materials are also crucial for students to comprehend Arabic Rhetoric lessons more effectively. According to Muhammad et al. (2006), textbook plays minimal assistance towards students' understanding of the Arabic Rhetoric subject. Samah (2006) and Abdullah (2008) found that students were lacking external materials and they perceived teaching aids that utilize the use of computer technology as more applicable and effective. Such argument thus gives support on the potential of teaching materials via computer technology to enhance students' performance in learning Arabic Rhetoric.

In view of the points highlighted, students are less interested and less motivated in learning Arabic Rhetoric. This paper believes that the problems mentioned above can be overcome with the use of technology in the teaching and learning process. This is in line with Hamid (2012) who designed and developed the prototype of 'Balaghah Arab' (Arabic Rhetoric) and found that the technology-based balaghah learning was effective and satisfying. Therefore, a research needs to be conducted to design and develop an application with improved quality and efficacy to ease students' learning so that they will be able to better understand and subsequently apply balaghah (Arabic Rhetoric) in their communication.

\section{Research Objectives}

The objectives of the study are as follows:

1- To identify the learning approach of Arabic Rhetoric subject among form four students.

2- To understand issues in Arabic Rhetorical learning approach and innovation tendencies among form four students.

3- To discover the importance of innovation and the design needed in learning Arabic Rhetoric among form four students. 


\section{Methodology}

A Needs Analysis survey was conducted in this study to investigate the students' needs related to the Arabic Rhetoric application. The purposive sampling comprised of 40 form four students from Sekolah Agama Menengah Tengku Ampuan Jemaah, Shah Alam. The instrument employed was a questionnaire comprising four parts, namely Part A (demographic information), Part B (learning approach of Arabic Rhetoric subject), Part C (issues in Arabic Rhetorical learning), and Part $D$ (innovation design in Arabic Rhetoric learning). The questionnaire used a five-point Likert scale ranging from strongly disagree (SD), disagree (D), uncertain (U), agree (A), and strongly agree (SA).

Prior to the study, the questionnaire was verified by an expert who checked the accurateness and validity of each item and construct. It was then distributed to the respondents via the Google Form application. The result showed that the reliability of this survey recorded 0.931 which is categorized as excellent (Gliem \& Gliem, 2003). The data were analyzed with frequency, percentage, mean (M) and standard deviation (SD) through descriptive statistics by employing the SPSS software version 26. The interpretation of mean score was done following Nunnally and Bernstein (1994) as illustrated in Table 1 below.

\section{Table 1}

Mean Score Interpretation

\begin{tabular}{cc}
\hline Mean score & Level \\
\hline $1.00-2.00$ & Low \\
\hline $2.01-3.00$ & Medium low \\
\hline $3.01-4.00$ & Medium \\
\hline $4.00-5.00$ & High \\
\hline
\end{tabular}

Note. Nunnally and Bernstein (1994)

The analysis serves the purpose of determining the composition and demographic characteristics of the respondents such as gender, results of PT3 Arabic subject, and level of ICT skill.

\section{Needs Analysis}

According to McKillip (1987), needs analysis is a process of identifying issues and determining solutions for a targeted audience. Information about the problems should be gathered so that it could be identified solved successfully (p. 20). The aim of conducting a needs analysis in this study is to investigate the existing issues and the need to develop an Arabic Rhetoric application. This was achieved through the survey technique where a questionnaire was administered to the respondents in eliciting their feedback on the need to develop an Arabic Rhetoric application. The design of the application should be practical in attracting students' motivation to the Arabic Rhetoric subject through MOOC. 


\section{Constructs and Items}

Table 2: Constructs and items

\begin{tabular}{|c|c|c|}
\hline & $\begin{array}{l}\text { Cronbach's } \\
\text { Alfa }\end{array}$ & $\begin{array}{c}\text { Number of } \\
\text { items }\end{array}$ \\
\hline \multicolumn{3}{|l|}{ A: Demography } \\
\hline \multicolumn{3}{|l|}{ Gender } \\
\hline \multicolumn{3}{|l|}{ Result of PT3 Arabic subject } \\
\hline \multicolumn{3}{|l|}{ Level of ICT skill } \\
\hline \multicolumn{3}{|l|}{ B: Arabic Rhetoric learning approach } \\
\hline Usage of Arabic Rhetoric learning materials. & .788 & 8 \\
\hline C: Issues in Arabic Rhetoric learning & .867 & 16 \\
\hline Issues in Arabic Rhetoric learning approach. & .802 & 8 \\
\hline $\begin{array}{l}\text { Issues of innovation tendency in Arabic Rhetoric } \\
\text { learning. }\end{array}$ & .883 & 8 \\
\hline D: Design innovation in Arabic Rhetoric learning & .929 & 19 \\
\hline $\begin{array}{l}\text { The importance of innovation in Arabic Rhetoric } \\
\text { learning. }\end{array}$ & .931 & 8 \\
\hline $\begin{array}{l}\text { Arabic Rhetoric application content design and } \\
\text { presentation. }\end{array}$ & .862 & 11 \\
\hline OVERALL & 0.931 & 43 \\
\hline
\end{tabular}

\section{Results and Findings}

A. Demographic Information

Table 3 describes the distribution of respondents' demography which comprises their gender, result of PT3 Arabic subject, and level of ICT skill.

Table 3: Demography of respondents.

\begin{tabular}{llcc}
\hline \multicolumn{1}{c}{ Item } & \multicolumn{1}{c}{ Category } & Frequency & Percentage (\%) \\
\hline Gender & Male & 18 & 45.0 \\
& Female & 22 & 55.0 \\
\hline Result of PT3 Arabic & $\mathrm{A}=$ (Excellent) & 24 & 60.0 \\
subject & $\mathrm{B}=$ (Good) & 10 & 25.0 \\
& $\mathrm{C}=$ (Medium) & 6 & 15.0 \\
\hline Level of ICT skill & No skill (never use ICT) & 1 & 2.5 \\
& Medium (rarely use ICT) & 15 & 37.5 \\
& Proficient (always use ICT) & 21 & 52.5 \\
& Very proficient (able to & & \\
& produce digital materials using & 3 & 7.5 \\
& ICT) & & \\
& & & \\
\end{tabular}




\section{INTERNATIONAL JOURNAL OF ACADEMIC RESEARCH IN PROGRESSIVE EDUCATION AND DEVELOPMENT \\ Vol. 10, No. 3, 2021, E-ISSN: 2226-6348 @ 2021 HRMARS}

The results indicate that the respondents' distribution by gender is almost equal. In terms of the respondents' PT3 result for the Arabic subject, the highest percentage is 'excellent' $(60 \%)$, followed by 'good' (25\%), and 'medium' (15\%). Meanwhile, almost all respondents are ICT literate with the majority of them are 'proficient' (always use ICT) (52.5\%), followed by 'medium' (rarely use ICT) (37.5\%), 'very proficient' (able to produce digital materials using ICT) $(7.5 \%)$, and only one respondent (2.5\%) is ICT illiterate.

\section{B: Arabic Rhetoric Learning Approach}

Table 4 contains results about the use of Arabic Rhetoric learning materials. It provides information on how students learn Arabic Rhetoric, whether by using textbooks, additional reference books, library materials, internet materials, and other resources.

Table 4: The use of Arabic Rhetoric learning materials.

\begin{tabular}{|c|c|c|c|c|c|c|c|c|c|}
\hline No & Item & SD & D & $\mathbf{U}$ & A & SA & $\mathbf{M}$ & SD & Interpretation \\
\hline B1 & $\begin{array}{l}\text { I use a } \\
\text { textbook. }\end{array}$ & $\begin{array}{c}0 \\
(0.0)\end{array}$ & $\begin{array}{c}0 \\
(0.0)\end{array}$ & $\begin{array}{c}5 \\
(12.5)\end{array}$ & $\begin{array}{c}19 \\
(47.5)\end{array}$ & $\begin{array}{c}16 \\
(40.0)\end{array}$ & 4.27 & .679 & High \\
\hline B2 & $\begin{array}{l}\text { I use additional } \\
\text { reference } \\
\text { books. }\end{array}$ & $\begin{array}{c}0 \\
(0.0)\end{array}$ & $\begin{array}{c}4 \\
(10.0)\end{array}$ & $\begin{array}{c}12 \\
(30.0)\end{array}$ & $\begin{array}{c}16 \\
(40.0)\end{array}$ & $\begin{array}{c}8 \\
(20.0)\end{array}$ & 3.70 & .911 & Medium \\
\hline B3 & $\begin{array}{l}\text { I use library } \\
\text { materials. }\end{array}$ & $\begin{array}{c}0 \\
(0.0)\end{array}$ & $\begin{array}{c}6 \\
(15.0)\end{array}$ & $\begin{array}{c}26 \\
(65.0)\end{array}$ & $\begin{array}{c}7 \\
(17.5)\end{array}$ & $\begin{array}{c}1 \\
(2.5)\end{array}$ & 3.08 & .656 & Medium \\
\hline B4 & $\begin{array}{l}\text { I use internet } \\
\text { materials. }\end{array}$ & $\begin{array}{c}1 \\
(2.5)\end{array}$ & $\begin{array}{c}3 \\
(7.5) \\
\end{array}$ & $\begin{array}{c}14 \\
(35.0) \\
\end{array}$ & $\begin{array}{c}16 \\
(40.0) \\
\end{array}$ & $\begin{array}{c}6 \\
(15.0) \\
\end{array}$ & 3.58 & .931 & Medium \\
\hline B5 & $\begin{array}{l}\text { I use short } \\
\text { notes made by } \\
\text { myself }\end{array}$ & $\begin{array}{c}1 \\
(2.5)\end{array}$ & $\begin{array}{c}1 \\
(2.5)\end{array}$ & $\begin{array}{c}5 \\
(12.5)\end{array}$ & $\begin{array}{c}24 \\
(60.0)\end{array}$ & $\begin{array}{c}9 \\
(22.5)\end{array}$ & 3.97 & .832 & Medium \\
\hline B6 & $\begin{array}{l}\text { I use computer } \\
\text { software to } \\
\text { learn. }\end{array}$ & $\begin{array}{c}1 \\
(2.5)\end{array}$ & $\begin{array}{c}7 \\
(17.5)\end{array}$ & $\begin{array}{c}19 \\
(47.5)\end{array}$ & $\begin{array}{c}12 \\
(30.0)\end{array}$ & $\begin{array}{c}1 \\
(2.5)\end{array}$ & 3.12 & .822 & Medium \\
\hline B7 & $\begin{array}{l}\text { I use a } \\
\text { summary of } \\
\text { graphical } \\
\text { notes. }\end{array}$ & $\begin{array}{c}1 \\
(2.5)\end{array}$ & $\begin{array}{c}5 \\
(12.5)\end{array}$ & $\begin{array}{c}13 \\
(32.5)\end{array}$ & $\begin{array}{c}14 \\
(35.0)\end{array}$ & $\begin{array}{c}7 \\
(17.5)\end{array}$ & 3.53 & 1.012 & Medium \\
\hline B8 & $\begin{array}{l}\text { I use notes } \\
\text { shared by } \\
\text { lecturers/teach } \\
\text { ers. }\end{array}$ & $\begin{array}{c}0 \\
(0.0)\end{array}$ & $\begin{array}{c}3 \\
(7.5)\end{array}$ & $\begin{array}{c}7 \\
(17.5)\end{array}$ & $\begin{array}{c}18 \\
(45.0)\end{array}$ & $\begin{array}{c}12 \\
(30.0)\end{array}$ & 3.97 & .891 & Medium \\
\hline & Overall & & & & & & 3.65 & .539 & Medium \\
\hline
\end{tabular}

$\mathrm{N}=40 \quad \mathrm{M}=$ Mean $\quad \mathrm{SD}=$ Standard Deviation

SD: Strongly Disagree D: Disagree U: Uncertain $\quad$ A: Agree $\quad$ SA: Strongly Agree Data in Table 4 show that the use of learning materials among the respondents is at a medium level, with an overall mean value of 3.65 and a standard deviation of .539. This indicates a moderate usage of learning materials by students to support their learning. The learning material with the highest mean score of usage is textbook $(M=4.27, S D=.679)$ followed by notes shared by lecturers $(M=3.97, S D=.891)$, short notes made by students $(M=3.97, S D$ $=.832)$, additional reference books $(M=3.70, S D=.911)$, internet materials $(M=3.58, S D=$ 


\section{INTERNATIONAL JOURNAL OF ACADEMIC RESEARCH IN PROGRESSIVE EDUCATION AND \\ DEVELOPMENT \\ Vol. 10, No. 3, 2021, E-ISSN: 2226-6348 @ 2021 HRMARS}

$.931)$, and graphical notes summary $(M=3.53, S D=1.012)$, computer software $(M=3.12, S D$ $=.822)$, and library materials $(M=3.08, S D=.656)$. All items have recorded mean values of medium and high. This concludes that the majority of respondents used textbook as their learning material to learn Arabic Rhetoric.

\section{C: Issues on Arabic Rhetoric Learning Approach}

This section attempts to uncover two issues pertaining to Arabic Rhetoric learning approach (see Table 5) and innovation tendency in Arabic Rhetoric learning (see Table 6).

Table 5: Arabic Rhetoric learning approach.

\begin{tabular}{|c|c|c|c|c|c|c|c|c|c|}
\hline No & Item & SD & D & $\mathbf{U}$ & A & SA & $\mathbf{M}$ & SD & Interpretation \\
\hline Ca1 & $\begin{array}{l}\text { Traditional } \\
\text { teaching (total } \\
\text { explanation by } \\
\text { the teacher) is } \\
\text { insufficient to } \\
\text { understand the } \\
\text { content of the } \\
\text { subject. }\end{array}$ & $\begin{array}{c}3 \\
(7.5)\end{array}$ & $\begin{array}{c}9 \\
(22.5)\end{array}$ & $\begin{array}{c}11 \\
(27.5)\end{array}$ & $\begin{array}{c}12 \\
(30.0)\end{array}$ & $\begin{array}{c}5 \\
(12.5)\end{array}$ & 3.18 & 1.152 & Medium \\
\hline $\mathrm{Ca} 2$ & $\begin{array}{l}\text { Difficulty to } \\
\text { communicate } \\
\text { with teachers } \\
\text { outside the } \\
\text { classroom. }\end{array}$ & $\begin{array}{c}4 \\
(10.0)\end{array}$ & $\begin{array}{c}6 \\
(15.0)\end{array}$ & $\begin{array}{c}11 \\
(27.5)\end{array}$ & $\begin{array}{c}16 \\
(40.0)\end{array}$ & $\begin{array}{c}3 \\
(7.5)\end{array}$ & 3.20 & 1.114 & Medium \\
\hline Ca3 & $\begin{array}{l}\text { Limited time to } \\
\text { delve into the } \\
\text { topics in class. }\end{array}$ & $\begin{array}{c}2 \\
(5.0)\end{array}$ & $\begin{array}{c}5 \\
(12.5)\end{array}$ & $\begin{array}{c}7 \\
(17.5)\end{array}$ & $\begin{array}{c}18 \\
(45.0)\end{array}$ & $\begin{array}{c}8 \\
(20.0)\end{array}$ & 3.62 & 1.102 & Medium \\
\hline Ca4 & $\begin{array}{l}\text { Syllabus content } \\
\text { is too much. }\end{array}$ & $\begin{array}{c}2 \\
(5.0) \\
\end{array}$ & $\begin{array}{c}9 \\
(22.5) \\
\end{array}$ & $\begin{array}{c}7 \\
(17.5) \\
\end{array}$ & $\begin{array}{c}16 \\
(40.0) \\
\end{array}$ & $\begin{array}{c}6 \\
(15.0) \\
\end{array}$ & 3.38 & 1.148 & Medium \\
\hline Ca5 & $\begin{array}{l}\text { Complex teaching } \\
\text { notes. }\end{array}$ & $\begin{array}{c}1 \\
(2.5)\end{array}$ & $\begin{array}{c}3 \\
(7.5) \\
\end{array}$ & $\begin{array}{c}9 \\
(22.5)\end{array}$ & $\begin{array}{c}22 \\
(55.0)\end{array}$ & $\begin{array}{c}5 \\
(12.5) \\
\end{array}$ & 3.68 & .888 & Medium \\
\hline Ca6 & $\begin{array}{l}\text { Lack of online } \\
\text { materials on the } \\
\text { internet. }\end{array}$ & $\begin{array}{c}2 \\
(5.0)\end{array}$ & $\begin{array}{c}7 \\
(17.5)\end{array}$ & $\begin{array}{c}14 \\
(35.0)\end{array}$ & $\begin{array}{c}13 \\
(32.5)\end{array}$ & $\begin{array}{c}4 \\
(10.0)\end{array}$ & 3.25 & 1.032 & Medium \\
\hline Ca7 & $\begin{array}{l}\text { High cost of } \\
\text { obtaining } \\
\text { additional } \\
\text { reference } \\
\text { materials. }\end{array}$ & $\begin{array}{c}0 \\
(0.0)\end{array}$ & $\begin{array}{c}11 \\
(27.5)\end{array}$ & $\begin{array}{c}18 \\
(45.0)\end{array}$ & $\begin{array}{c}6 \\
(15.0)\end{array}$ & $\begin{array}{c}5 \\
(12.5)\end{array}$ & 3.12 & .966 & Medium \\
\hline $\mathrm{Ca} 8$ & $\begin{array}{l}\text { Unavailability of } \\
\text { completed } \\
\text { teaching aids. }\end{array}$ & $\begin{array}{c}2 \\
(5.0)\end{array}$ & $\begin{array}{c}9 \\
(22.5)\end{array}$ & $\begin{array}{c}20 \\
(50.0)\end{array}$ & $\begin{array}{c}7 \\
(17.5)\end{array}$ & $\begin{array}{c}2 \\
(5.0)\end{array}$ & 2.95 & .904 & $\begin{array}{l}\text { Medium } \\
\text { Low }\end{array}$ \\
\hline & Overall & & & & & & 3.30 & .675 & Medium \\
\hline
\end{tabular}




\section{INTERNATIONAL JOURNAL OF ACADEMIC RESEARCH IN PROGRESSIVE EDUCATION AND DEVELOPMENT \\ Vol. 10, No. 3, 2021, E-ISSN: 2226-6348 @ 2021 HRMARS}

Table 5 above shows the data on Arabic Rhetoric learning approach, which recorded a moderate level with a mean score of 3.30 and a standard deviation of .675. All items obtained a mean average score at a medium level, with the exception of "unavailability of completed teaching aids" which recorded a medium low level $(M=2.95, S D=0.904)$. The highest mean score was recorded by "complex teaching notes" $(M=3.68, S D=0.888)$ followed by "limited time to delve into the topics in class" ( $M=3.62, S D=1.102)$. Whereas other items in this constrict had recorded medium low to medium results, namely "syllabus content is too much." ( $M=3.38, S D=1.148)$, "lack of online materials on the internet" ( $M=3.25,1.032)$, "difficulty to communicate with teachers outside the classroom" ( $M=3.20, S D=1.114)$, "traditional teaching (total explanation by the teacher) is insufficient to understand the content of the subject." ( $M=3.18, S D=1.152)$, and "high cost of obtaining additional reference materials" ( $M=3.12, S D=.966)$. In summary, the unavailability of completed teaching aids reported in this result is in line with Muhammad et al. (2006) who stated that textbook has minimal assistance to students' understanding of Arabic Rhetoric in class. This has led Hanim (2014) to suggest on the need properly designed teaching materials that can effectively assist in achieving the learning objectives of an Arabic Rhetoric course.

Table 6: Innovation tendency in Arabic Rhetoric learning.

\begin{tabular}{|c|c|c|c|c|c|c|c|c|c|}
\hline No & Item & SD & D & U & A & SA & Mean & SD & Interpretation \\
\hline Cb1 & $\begin{array}{l}\text { I prefer learning } \\
\text { using multimedia } \\
\text { technology } \\
\text { application. }\end{array}$ & $\begin{array}{c}1 \\
(2.5)\end{array}$ & $\begin{array}{c}2 \\
(5.0)\end{array}$ & $\begin{array}{c}15 \\
(37.5)\end{array}$ & $\begin{array}{c}19 \\
(47.5)\end{array}$ & $\begin{array}{c}3 \\
(7.5)\end{array}$ & 3.52 & .816 & Medium \\
\hline $\mathrm{Cb} 2$ & $\begin{array}{l}\text { I love interactive } \\
\text { learning } \\
\text { materials. }\end{array}$ & $\begin{array}{c}1 \\
(2.5)\end{array}$ & $\begin{array}{c}0 \\
(0.0)\end{array}$ & $\begin{array}{c}7 \\
(17.5)\end{array}$ & $\begin{array}{c}26 \\
(65.0)\end{array}$ & $\begin{array}{c}6 \\
(15.0)\end{array}$ & 3.90 & .744 & Medium \\
\hline $\mathrm{Cb3}$ & $\begin{array}{l}\text { I prefer to learn a } \\
\text { subject using } \\
\text { pictorial } \\
\text { materials }\end{array}$ & $\begin{array}{c}0 \\
(0.0)\end{array}$ & $\begin{array}{c}0 \\
(0.0)\end{array}$ & $\begin{array}{c}7 \\
(17.5)\end{array}$ & $\begin{array}{c}17 \\
(42.5)\end{array}$ & $\begin{array}{c}16 \\
(40.0)\end{array}$ & 4.23 & .733 & High \\
\hline $\mathrm{Cb} 4$ & $\begin{array}{l}\text { I prefer to learn a } \\
\text { subject using } \\
\text { colored materials }\end{array}$ & $\begin{array}{c}1 \\
(2.5)\end{array}$ & $\begin{array}{c}0 \\
(0.0)\end{array}$ & $\begin{array}{c}2 \\
(5.0)\end{array}$ & $\begin{array}{c}15 \\
(37.5)\end{array}$ & $\begin{array}{c}22 \\
(55.0)\end{array}$ & 4.43 & .813 & High \\
\hline Cb5 & $\begin{array}{l}\text { I love reading } \\
\text { information with } \\
\text { intriguing } \\
\text { graphics. }\end{array}$ & $\begin{array}{c}1 \\
(2.5)\end{array}$ & $\begin{array}{c}1 \\
(2.5)\end{array}$ & $\begin{array}{c}4 \\
(10.0)\end{array}$ & $\begin{array}{c}13 \\
(32.5)\end{array}$ & $\begin{array}{c}21 \\
(52.5)\end{array}$ & 4.30 & .939 & High \\
\hline $\mathrm{Cb} 6$ & $\begin{array}{l}\text { I love } \\
\text { information } \\
\text { written with } \\
\text { attractive font. }\end{array}$ & $\begin{array}{c}1 \\
(2.5)\end{array}$ & $\begin{array}{c}1 \\
(2.5)\end{array}$ & $\begin{array}{c}7 \\
(17.5)\end{array}$ & $\begin{array}{c}13 \\
(32.5)\end{array}$ & $\begin{array}{c}18 \\
(45.0)\end{array}$ & 4.15 & .975 & High \\
\hline $\mathrm{Cb} 7$ & $\begin{array}{l}\text { I like information } \\
\text { presented with } \\
\text { animation } \\
\text { (moving). }\end{array}$ & $\begin{array}{c}1 \\
(2.5)\end{array}$ & $\begin{array}{c}3 \\
(7.5)\end{array}$ & $\begin{array}{c}11 \\
(27.5)\end{array}$ & $\begin{array}{c}12 \\
(30.0)\end{array}$ & $\begin{array}{c}13 \\
(32.5)\end{array}$ & 3.83 & 1.059 & Medium \\
\hline
\end{tabular}


INTERNATIONAL JOURNAL OF ACADEMIC RESEARCH IN PROGRESSIVE EDUCATION AND

DEVELOPMENT

Vol. 10, No. 3, 2021, E-ISSN: 2226-6348 @ 2021 HRMARS

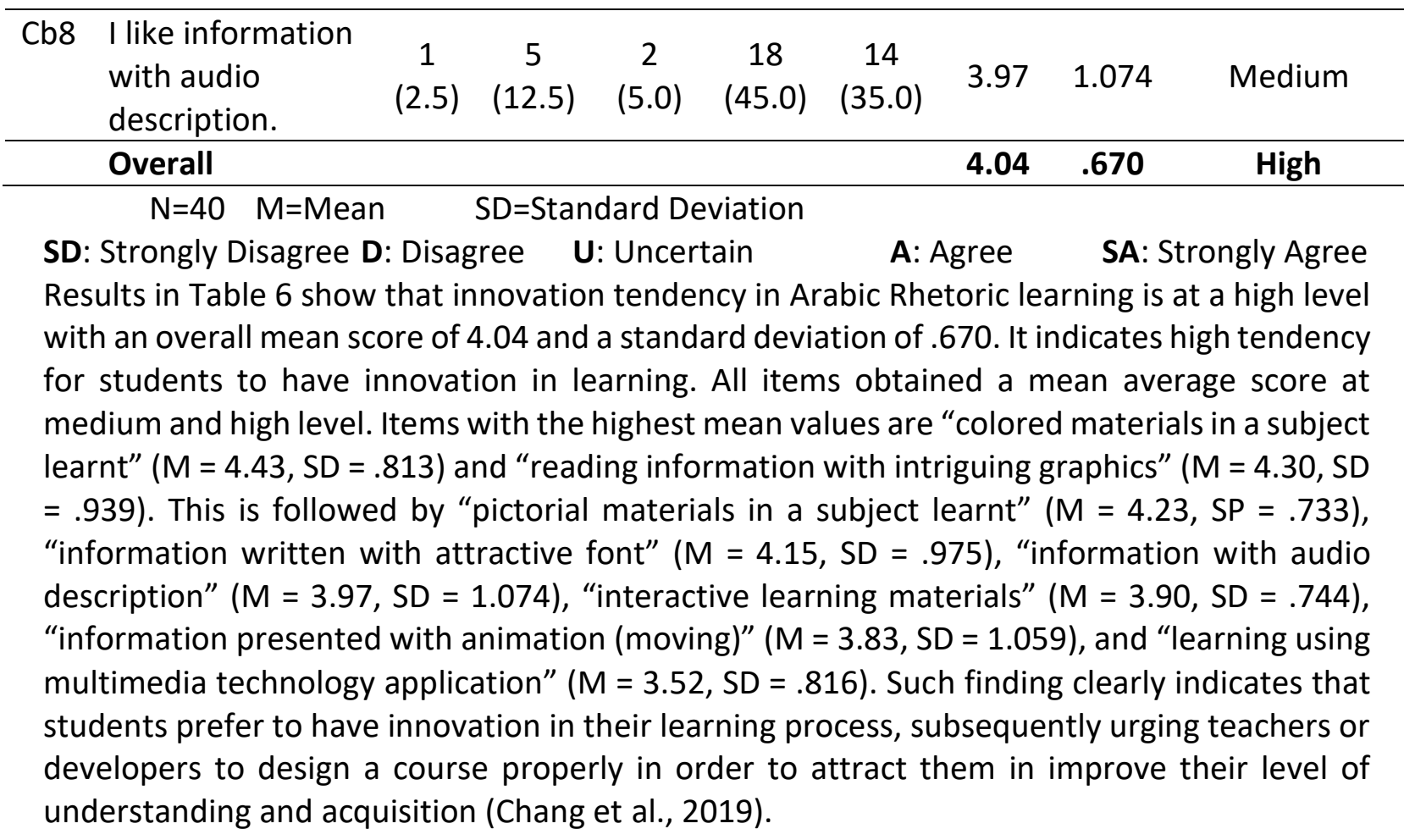

\section{D: Innovation Design in Arabic Rhetoric Learning}

This section investigates the importance of innovation in Arabic Rhetoric learning (see Table 7) and the content design and presentation of the Arabic Rhetoric application (see Table 8).

Table 7: The importance of innovation in Arabic rhetorical learning

\begin{tabular}{|c|c|c|c|c|c|c|c|c|c|}
\hline No & Item & SD & D & $\mathbf{U}$ & A & SA & Mean & SD & Interpretation \\
\hline Da1 & $\begin{array}{l}\text { I believe that } \\
\text { animated Arabic } \\
\text { Rhetoric } \\
\text { application makes } \\
\text { information } \\
\text { easier to } \\
\text { understand. }\end{array}$ & $\begin{array}{c}1 \\
(2.5)\end{array}$ & $\begin{array}{c}0 \\
(0.0)\end{array}$ & $\begin{array}{c}3 \\
(7.5)\end{array}$ & $\begin{array}{c}18 \\
(45.0)\end{array}$ & $\begin{array}{c}18 \\
(45.0)\end{array}$ & 4.30 & .823 & High \\
\hline $\mathrm{Da} 2$ & $\begin{array}{l}\text { I believe that } \\
\text { animated Arabic } \\
\text { Rhetorical } \\
\text { application makes } \\
\text { information more } \\
\text { concise. }\end{array}$ & $\begin{array}{c}1 \\
(2.5)\end{array}$ & $\begin{array}{c}1 \\
(2.5)\end{array}$ & $\begin{array}{c}5 \\
(12 . \\
5)\end{array}$ & $\begin{array}{c}19 \\
(47.5)\end{array}$ & $\begin{array}{c}14 \\
(35.0)\end{array}$ & 4.10 & .900 & High \\
\hline Da3 & $\begin{array}{l}\text { I believe that } \\
\text { animated videos } \\
\text { in the application } \\
\text { enhance thinking } \\
\text { creativity. }\end{array}$ & $\begin{array}{c}1 \\
(2.5)\end{array}$ & $\begin{array}{c}0 \\
(0.0)\end{array}$ & $\begin{array}{c}10 \\
(25 . \\
0)\end{array}$ & $\begin{array}{c}15 \\
(37.5)\end{array}$ & $\begin{array}{c}14 \\
(35.0)\end{array}$ & 4.03 & .920 & High \\
\hline Da4 & $\begin{array}{l}\text { I believe that } \\
\text { animated videos } \\
\text { in the application }\end{array}$ & $\begin{array}{c}1 \\
(2.5)\end{array}$ & $\begin{array}{c}2 \\
(5.0)\end{array}$ & $\begin{array}{c}12 \\
(30 . \\
0) \\
\end{array}$ & $\begin{array}{c}12 \\
(30.0)\end{array}$ & $\begin{array}{c}13 \\
(32.5)\end{array}$ & 3.85 & $\begin{array}{c}1.02 \\
7\end{array}$ & Medium \\
\hline
\end{tabular}




\begin{tabular}{|c|c|c|c|c|c|c|c|c|c|}
\hline & $\begin{array}{l}\text { make learning } \\
\text { more focused. }\end{array}$ & & & & & & & & \\
\hline Da5 & $\begin{array}{l}\text { I believe that } \\
\text { animated videos } \\
\text { in the application } \\
\text { make learning } \\
\text { faster. }\end{array}$ & $\begin{array}{c}1 \\
(2.5)\end{array}$ & $\begin{array}{c}1 \\
(2.5)\end{array}$ & $\begin{array}{c}11 \\
(27 . \\
5)\end{array}$ & $\begin{array}{c}15 \\
(37.5)\end{array}$ & $\begin{array}{c}12 \\
(30.0)\end{array}$ & 3.90 & .955 & Medium \\
\hline Da6 & $\begin{array}{l}\text { I believe that } \\
\text { animated videos } \\
\text { in the application } \\
\text { motivate } \\
\text { students to study } \\
\text { harder. }\end{array}$ & $\begin{array}{c}1 \\
(2.5)\end{array}$ & $\begin{array}{c}4 \\
(10.0)\end{array}$ & $\begin{array}{c}7 \\
(17 . \\
5)\end{array}$ & $\begin{array}{c}14 \\
(35.0)\end{array}$ & $\begin{array}{c}14 \\
(35.0)\end{array}$ & 3.90 & $\begin{array}{c}1.08 \\
1\end{array}$ & Medium \\
\hline Da7 & $\begin{array}{l}\text { I believe that } \\
\text { animated videos } \\
\text { in the application } \\
\text { make learning } \\
\text { more fun. }\end{array}$ & $\begin{array}{c}2 \\
(5.0)\end{array}$ & $\begin{array}{c}0 \\
(0.0)\end{array}$ & $\begin{array}{c}3 \\
(7.5)\end{array}$ & $\begin{array}{c}17 \\
(42.5)\end{array}$ & $\begin{array}{c}18 \\
(45.0)\end{array}$ & 4.23 & .974 & High \\
\hline \multirow[t]{3}{*}{ Da8 } & $\begin{array}{l}\text { I believe that } \\
\text { learning through } \\
\text { animated videos } \\
\text { is more effective. }\end{array}$ & $\begin{array}{c}1 \\
(2.5)\end{array}$ & $\begin{array}{c}0 \\
(0.0)\end{array}$ & $\begin{array}{c}19 \\
(47 . \\
5)\end{array}$ & $\begin{array}{c}13 \\
(32.5)\end{array}$ & $\begin{array}{c}7 \\
(17.5)\end{array}$ & 3.63 & .868 & Medium \\
\hline & Overall & & & & & & 3.99 & .777 & Medium \\
\hline & $\mathrm{N}=40 \quad \mathrm{M}=$ Mean & $S D=S$ & tandard & Deviatic & & & & & \\
\hline $\begin{array}{l}\text { SD: Str } \\
\text { Table } \\
\text { level v } \\
\text { constr } \\
\text { margir } \\
\text { subsec } \\
\text { level. } \\
\text { are “a } \\
4.30,5 \\
4.23 \text {, } \\
\text { inform } \\
\text { enhan } \\
\text { learnir } \\
\text { to stuc } \\
\text { more } \\
\text { effecti } \\
\text { to crea }\end{array}$ & 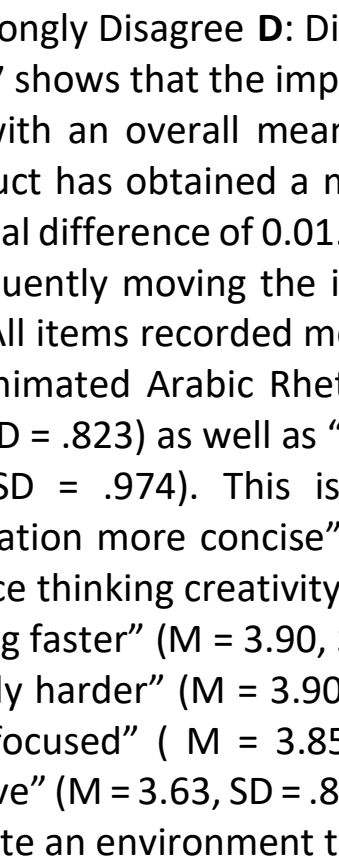 & $\begin{array}{l}\text { agree } \\
\text { rtance } \\
\text { score } \\
\text { oderat } \\
\text { The res } \\
\text { nportar } \\
\text { dium a } \\
\text { oric ap } \\
\text { nimate } \\
\text { follow } \\
\text { (M = } 4 \\
\text { (M = } 4 \\
D=.95 \\
\text { SD = } 1 \\
\text { SD = } \\
\text { 8). Suc } \\
\text { at will }\end{array}$ & $\begin{array}{l}\text { U: Un } \\
\text { of innov } \\
\text { of } 3.99 \\
\text { mean } \\
\text { earcher } \\
\text { ice of ir } \\
\text { id high } \\
\text { lication } \\
\text { d video } \\
\text { d by " } \\
10, \text { SD } \\
.03, \text { SD } \\
\text { 5), " anir } \\
\text { 081), “ } \\
1.027) \text {, } \\
\text { finding } \\
\text { oster st }\end{array}$ & $\begin{array}{l}\text { ertain } \\
\text { ation in } \\
\text { and a s } \\
\text { core, t } \\
\text { perceiv } \\
\text { covatio } \\
\text { nean sc } \\
\text { makes } \\
\text { in the } \\
\text { animate } \\
.900), \\
.920), \\
\text { lated vi } \\
\text { nimate } \\
\text { nd “lea } \\
\text { thus su } \\
\text { idents' }\end{array}$ & $\begin{array}{l}\text { Arabic } \\
\text { tandard } \\
\text { he figure } \\
\text { ed that } \\
\text { n in Ara } \\
\text { ores. Ite } \\
\text { informa } \\
\text { applicati } \\
\text { d Arab } \\
\text { "animat } \\
\text { "animat } \\
\text { deos in t } \\
\text { d videos } \\
\text { irning th } \\
\text { ggests th } \\
\text { interest }\end{array}$ & $\begin{array}{l}\text { A: Agree } \\
\text { hetorical } \\
\text { deviatior } \\
\text { is close } \\
\text { he figure } \\
\text { bic Rhetc } \\
\text { ms with } \\
\text { tion easi } \\
\text { on make } \\
\text { c Rheto } \\
\text { ed video } \\
\text { ed videos } \\
\text { he applic } \\
\text { in the ap } \\
\text { irough al } \\
\text { at innova } \\
\text { (Hat, } 201\end{array}$ & $\begin{array}{l}\text { Sf } \\
\text { learnin } \\
\text { of .77 } \\
\text { o the } \\
\text { can be } \\
\text { ic lear } \\
\text { e high } \\
r \text { to ut } \\
\text { earnin } \\
\text { cal ap } \\
\text { in the } \\
n \text { the } \\
\text { tion } m \\
\text { olicatic } \\
\text { imatec } \\
\text { ion in } \\
\text { ). }\end{array}$ & $\begin{array}{l}\text { Stron } \\
\text { is at a } \\
\text { Althe } \\
\text { gh lev } \\
\text { ounde } \\
\text { ing to } \\
\text { st me } \\
\text { derstar } \\
\text { more } \\
\text { licatio } \\
\text { pplica } \\
\text { oplicat } \\
\text { tivate } \\
\text { make } \\
\text { videos } \\
\text { arning }\end{array}$ & $\begin{array}{l}\text { Agree } \\
\text { dium } \\
\text { this } \\
\text { vith a } \\
4.00 \text {, } \\
\text { high } \\
\text { cores } \\
\text { (M = } \\
\text { ( } \mathrm{M}= \\
\text { nakes } \\
\text { may } \\
\text { make } \\
\text { dents } \\
\text { rning } \\
\text { more } \\
\text { rucial }\end{array}$ \\
\hline
\end{tabular}


INTERNATIONAL JOURNAL OF ACADEMIC RESEARCH IN PROGRESSIVE EDUCATION AND

DEVELOPMENT

Vol. 10, No. 3, 2021, E-ISSN: 2226-6348 @ 2021 HRMARS

Table 8: Content design and presentation of the Arabic Rhetoric application.

\begin{tabular}{|c|c|c|c|c|c|c|c|c|c|}
\hline No & Item & ST & D & U & A & SA & Mean & SD & Interpretation \\
\hline Db1 & $\begin{array}{l}\text { Learning } \\
\text { outcomes for } \\
\text { each topic } \\
\text { should be in } \\
\text { the form of a } \\
\text { concise text. }\end{array}$ & $\begin{array}{c}0 \\
(0.0)\end{array}$ & $\begin{array}{c}1 \\
(2.5)\end{array}$ & $\begin{array}{c}2 \\
(5.0)\end{array}$ & $\begin{array}{c}23 \\
(57.5)\end{array}$ & $\begin{array}{c}14 \\
(35.0)\end{array}$ & 4.25 & .670 & High \\
\hline Db2 & $\begin{array}{l}\text { Description of } \\
\text { video content } \\
\text { is necessary to } \\
\text { ease } \\
\text { understanding. }\end{array}$ & $\begin{array}{c}0 \\
(0.0)\end{array}$ & $\begin{array}{c}0 \\
(0.0)\end{array}$ & $\begin{array}{c}6 \\
(15.0)\end{array}$ & $\begin{array}{c}16 \\
(40.0)\end{array}$ & $\begin{array}{c}18 \\
(45.0)\end{array}$ & 4.30 & .723 & High \\
\hline Db3 & $\begin{array}{l}\text { Each topic } \\
\text { should be } \\
\text { wrapped up } \\
\text { with a drill } \\
\text { exercise. }\end{array}$ & $\begin{array}{c}0 \\
(0.0)\end{array}$ & $\begin{array}{c}0 \\
(0.0)\end{array}$ & $\begin{array}{c}5 \\
(12.5)\end{array}$ & $\begin{array}{c}10 \\
(25.0)\end{array}$ & $\begin{array}{c}25 \\
(62.5)\end{array}$ & 4.50 & .716 & High \\
\hline Db4 & $\begin{array}{l}\text { The choice of } \\
\text { moving font } \\
\text { should be easy } \\
\text { to read. }\end{array}$ & $\begin{array}{c}0 \\
(0.0)\end{array}$ & $\begin{array}{c}4 \\
(10.0)\end{array}$ & $\begin{array}{c}12 \\
(30.0)\end{array}$ & $\begin{array}{c}12 \\
(30.0)\end{array}$ & $\begin{array}{c}12 \\
(30.0)\end{array}$ & 3.80 & .992 & Medium \\
\hline Db5 & $\begin{array}{l}\text { The choice of } \\
\text { font color, font } \\
\text { size, icons, and } \\
\text { graphics } \\
\text { should be } \\
\text { appropriate. }\end{array}$ & $\begin{array}{c}0 \\
(0.0)\end{array}$ & $\begin{array}{c}0 \\
(0.0)\end{array}$ & $\begin{array}{c}1 \\
(2.5)\end{array}$ & $\begin{array}{c}14 \\
(35.0)\end{array}$ & $\begin{array}{c}25 \\
(62.5)\end{array}$ & 4.60 & .545 & High \\
\hline Db6 & $\begin{array}{l}\text { Informative } \\
\text { graphics are } \\
\text { necessary to } \\
\text { provide a good } \\
\text { understanding. }\end{array}$ & $\begin{array}{c}0 \\
(0.0)\end{array}$ & $\begin{array}{c}0 \\
(0.0)\end{array}$ & $\begin{array}{c}3 \\
(7.5)\end{array}$ & $\begin{array}{c}15 \\
(37.5)\end{array}$ & $\begin{array}{c}22 \\
(55.0)\end{array}$ & 4.48 & .640 & High \\
\hline Db7 & $\begin{array}{l}\text { Audios need to } \\
\text { be clearly } \\
\text { pronounced. }\end{array}$ & $\begin{array}{c}0 \\
(0.0)\end{array}$ & $\begin{array}{c}1 \\
(2.5)\end{array}$ & $\begin{array}{c}0 \\
(0.0)\end{array}$ & $\begin{array}{c}13 \\
(32.5)\end{array}$ & $\begin{array}{c}26 \\
(65.0)\end{array}$ & 4.60 & .632 & High \\
\hline Db8 & $\begin{array}{l}\text { Videos need } \\
\text { appropriate } \\
\text { quality. }\end{array}$ & $\begin{array}{c}0 \\
(0.0)\end{array}$ & $\begin{array}{c}0 \\
(0.0)\end{array}$ & $\begin{array}{c}1 \\
(2.5)\end{array}$ & $\begin{array}{c}12 \\
(30.0)\end{array}$ & $\begin{array}{c}27 \\
(67.5)\end{array}$ & 4.65 & .533 & High \\
\hline Db9 & $\begin{array}{l}\text { The layout } \\
\text { should be } \\
\text { attractive. }\end{array}$ & $\begin{array}{c}0 \\
(0.0)\end{array}$ & $\begin{array}{c}0 \\
(0.0)\end{array}$ & $\begin{array}{c}4 \\
(10.0)\end{array}$ & $\begin{array}{c}12 \\
(30.0)\end{array}$ & $\begin{array}{c}24 \\
(60.0)\end{array}$ & 4.50 & .679 & High \\
\hline Db10 & $\begin{array}{l}\text { I should have } \\
\text { the option of } \\
\text { asking the } \\
\text { facilitator in }\end{array}$ & $\begin{array}{c}0 \\
(0.0)\end{array}$ & $\begin{array}{c}0 \\
(0.0)\end{array}$ & $\begin{array}{c}5 \\
(12.5)\end{array}$ & $\begin{array}{c}20 \\
(50.0)\end{array}$ & $\begin{array}{c}15 \\
(37.5)\end{array}$ & 4.25 & .670 & \\
\hline
\end{tabular}




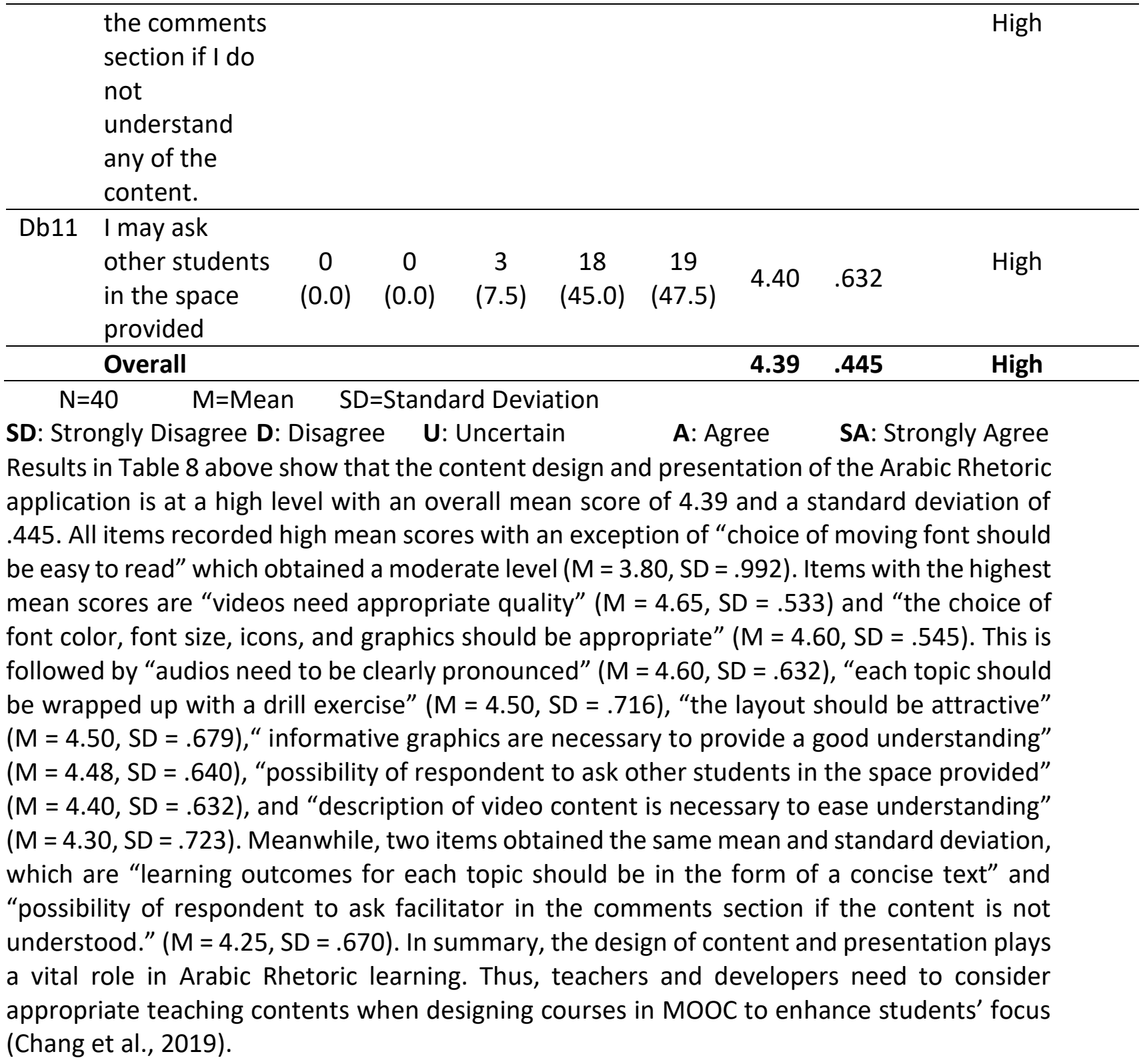

\section{Discussion of Results and Findings}

The first objective of this study is to identify the learning approach used by form four students to learn the Arabic Rhetorical subject. Findings reported in this study indicate dominant use of textbook among students due to the fact it is the basic resource in understanding the subject. On the other hand, the use of library materials is not prominent among students probably due to the limited availability of materials.

Meanwhile, the second objective delves into the issues of Arabic Rhetoric learning approach and the tendency of innovation among form four students. It was found that the issue of complex teaching notes is a main concern among students as it distorts students' learning and understanding to the Arabic Rhetorical contents. This finding is in line with Zaki et al. (2019), Ibrahim et al. (2019), and Hasan (2014) where the tendency of innovation revealed that students like colored materials in learning. Moreover, colored presentations can be created in the form of animated videos or animated infographic (Hamid et al., 2020). It plays a vital role in sustaining students' attention and aids their brain to have longitudinal memory of the 
lessons. The tendency of innovation in learning seems to be noticeable as its average mean score was recorded as high. Thus, it is safe to conclude that an Arabic Rhetoric application with multimedia elements will be positively received by students.

Finally, the third objective is related to the importance of innovation and design in learning Arabic Rhetoric. The majority of respondents believe that the animated application makes information easier to be conceived and learning process to become more fun. In addition, videos that incorporate font color, size, icon, and graphic display must be in good quality so that it can be viewed clearly and does not disrupt students' concentration during the learning process. This finding is aligned with past literature which suggests interactive as an essential element to promote high motivation among students (Redzaudin et al., 2021).

\section{Conclusion}

In conclusion, the current study has found that the application of Arabic Rhetoric course is needed by students to promote interesting self-learning process in the form of online application with multimedia elements such as videos, audios, graphics, images, animation, and interactivity.

The construct of content design and presentation of the Arabic Rhetoric application has recorded positive response compared to other constructs. The finding is in line with a number of past studies adopting multimedia applications (Sardi, 2009; Sahrir, 2011; Zulkhairi, 2012; Zainuddin, 2014; Yahya, 2016; Puasa, 2015).

Following these findings, there is a need to address and overcome the problems associated with Arabic Rhetoric learning encountered by students. Thus, the application must be properly designed and developed to meet the students' needs in the learning process. If well utilized, this form of e-learning will benefit students and motivates them to delve into the lessons positively. Additionally, innovation factors such as animation and video quality video also contribute to a more meaningful learning.

\section{Motivation and Contribution}

The Arabic Rhetorical application is seen to have the ability to increase the motivation of generation $Y$ students since it includes engaging multimedia components such as interactive and animated elements, as well as colourful learning materials. In addition, the online application will include comprehensive teaching and learning materials such as topic description videos, notes, activities, reinforcement exercises, and so on. As a result, it can assist teachers in the process of providing teaching aids.

\section{References}

Abdullah, A. H., \& Omar, M. C. (2016). A prolonged dilemma: Traditional curriculum of Arabic rhetoric (Balaghah) in Malaysia. Medwell Journals. The Social Sciences 11(6), 10081014.

Abdullah, A. H. (2008). Pengajaran balaghah peringkat STPM di sekolah-Sekolah menengah kebangsaan agama (SMKA). Jurnal Islam Dan Masyarakat Kontemporari, 1(1), 83-97.

Redzaudin, A. G., Rofian, M. I., Akmar, K. A. L., Adnan, M. A., \& Muhammad, D. (2016). Analisis Keperluan Pembangunan Modul Balaghah Bagi Pelajar Sarjana Muda Bahasa Arab Sebagai Bahasa Kedua. In International Conference on Aqidah, Dakwah and Syariah 2016 (IRSYAD 2016) (pp. 261-268). https://docplayer.net/68599397-Analisis- 
DEVELOPMENT

Vol. 10, No. 3, 2021, E-ISSN: 2226-6348 @ 2021 HRMARS

keperluan-pembangunan-modul-balaghah-bagi-pelajar-sarjana-muda-bahasa-arabsebagai-bahasa-kedua.html

Ariffin, A. H. (1999). Pengajaran Ilmu Balaghah (Retorika) di sekolah Menengah Kebangsaan Agama di Bawah Kementerian Pendidikan. Jabatan Pengajian Arab dan Tamadun Islam, Fakulti Pengajian Islam, Universiti Kebangsaan Malaysia, Bangi.

Bahagian Pembangunan Kurikulum KBD. (2016). Dokumen Standard Kurikulum al-Adab wa alBalaghah Tingkatan 5. Kementerian Pendidikan Malaysia.

Baharun, M. N. A. S., Imani, N., Haqqi, M. (2017). الأدب والبلاغة للسنة الرابعة. Aras Mega Sdn. Bhd: Kajang.

Chang, J., J., Lin, W. S., \& Chen, H. R. (2019). How attention level and cognitive style affect learning in a MOOC environment? Based on the perspective of brainwave analysis. Computers in Human Behavior 100, 209-217. https://doi.org/10.1016/j.chb.2018.08.016.

Hamid, M. Z. A. (2012). Reka bentuk perisian pembelajaran retorika Arab peringkat Sekolah Menengah [Unpublished master dissertation]. Universiti Teknologi MARA (UiTM).

Hanim, S., Rouyan, N. M., Anas, M., \& Yunus, K. (2014). Kepentingan memperkenalkan model strategi pengajaran balaghah peringkat IPT di Malaysia. Seminar Pengajaran dan Pembelajaran Bahasa Arab 2014, 14 September 2014, Auditorium Blok Akademik UniSZA.http://eprints.unisza.edu.my/id/eprint/491.

Hasan, E. A. (2014). Penggunaan peta minda dalam pembelajaran balaghah di sekolah menengah kebangsaan agama Tun Perak, Melaka, Malaysia [Unpublished master dissertation]. Universiti Putra Malaysia.

Hat, N. C., Sha'ari, S. H., Hamid, M. F. A. (2013). Persepsi pelajar terhadap penggunaan animasi dalam pembelajaran bahasa Arab. Jurnal Teknologi (Social Sciences) 63(1), 25-29. https://doi.org/10.11113/jt.v63.1683.

Ibrahim, A. A. (2014). Penguasaan haqiqah dan majaz dalam kalangan pelajar peringkat menengah [Master dissertation, Universiti Malaya]. UM Students Repository http://studentsrepo.um.edu.my/5406/

Ibrahim, A. A., Zaki, M., \& Azhar. (2019). Penguasaan Komponen Retorik Bahasa Arab dalam kalangan Pelajar Peringkat Sijil Pelajaran Malaysia (SDM). INSANIAH: Online Journal of Language, Communication, and Humanities, 2(2), 1-16.

McKillip, J. (1990). Need Analysis, Tools for the Human Services and Education, New Delhi: Sage Publications Inc. https://archive.org/details/needanalysistool0000mcki/page/n5/mode/2up.

Kamarudin, N., Tien, L.T., Sharif, A. H., Taha, H., Rahim, N. A. (2020). Pembangunan dan persepsi pelajar terhadap Modul e-Pentaksiran untuk Massive Open Online Course (MOOC) Kimia. Journal of Science and Mathematics Letters (8)2, 109-121. https://doi.org/10.37134/jsml.vol8.2.13.2020.

Korableva, O., Durand, T., Kalimulina, O., Stepanova, I. (2019). Studying user satisfaction with the mooc platform interfaces using the example of coursera and open education platforms. ICBDE'19: Proceedings of the 2019 International Conference on Big Data and Education, 26-30. https://doi.org/10.1145/3322134.3322139

Mohamad, N., Othman, A., Azaruddin, M. Z. S. N., Rajah, N., Isa, M. H. M. (2020). The performance of business school student using massive open online courses (MOOCs) at Universiti Teknikal Malaysia Melaka (UTeM). International Journal of Advanced Trends in Computer Science and Engineering (9)5. 
DEVELOPMENT

Vol. 10, No. 3, 2021, E-ISSN: 2226-6348 @ 2021 HRMARS

https://doi.org/10.30534/ijatcse/2020/259952020.

Muhammad, A., Abdullah, A. H., Basiron, B., Jasmi, K. A., Noor, S. S. M. (2006), Penguasaan Pelajar Sekolah Menengah Aliran Agama Terhadap Pengajian Ilmu Retorik Arab. Pusat Pengajian Islam dan Pembangunan Sosial. Universiti Teknologi Malaysia.

Muhammad, A., Mohamed, A. K., \& Jasmi, K. A. (2005). Sudut perbezaan mata pelajaran balaghah peringkat SPM dan STPM mempengaruhi perkaedahan dan teknik pengajaran dan pembelajaran. Wacana Pendidikan Islam Peringkat Kebangsaan [siri ketiga] at Hotel Alson Klana, Seremban on 16 October 2003.

Nunnally, J. C., \& Bernstein, I. H. (1994). Psychometric theory (3rd ed.). New York: McGrawHill.

Piech, C., Huang, J., Chen, Z. (2013). Tuned models of peer assessment in MOOCs. Proceedings of the 6th International Conference on Educational Data Mining (EDM 2013). http://arxiv.org/abs/1307.2579.

Puasa, B. (2015). Reka bentuk aplikasi subjek morfologi bahasa arab menggunakan animasi interaktif [Unpublished master dissertation]. Universiti Teknologi MARA (UiTM).

Qarabesh, M. A. M. (2020). Learning style preferences and attitudes to moocs: common grounds for Saudi EFL learners' success. Asian EFL Journal Research Articles (27)4.4. https://www.asian-efl-journal.com/monthly-editions-new/2020-monthlyeditions/volume-27-issue-4-4-october-2020.

Rahim, M. I., \& Shamsudin, S. (2019). Categorisation of video lecture designs in MOOC for technical and vocational education and training educators. Journal of Technical Education and Training (11) 4, 11-17. https://doi.org/10.30880/jtet.2019.11.04.002.

Sahrir, M. S. (2011). Analysis, design, and development of an online vocabulary game for arabic elementary learners [Unpublished doctoral dissertation]. Universiti Teknologi MARA (UiTM).

Samah, R., \& Ismail, M. M. (2006). Penguasaan 'Balaghah' dan permasalahannya di kalangan pelajar Sikil Tingi Agama Malaysia (STAM) (Kajian di sekolah-sekolah agama Negeri Selangor). Kolej Universiti Islam Malaysia. Bandar Baru Nilai. Negeri Sembilan.

Sardi, J. (2009). Pembelajaran nahu dalam bahasa arab komunikasi menggunakan aplikasi multimedia berasaskan web [Unpublished doctoral dissertation]. Universiti Malaya.

Sopian, A., Ahmad, S., \& Bakar, K. A. (2013). Penguasaan dan permasalahan pelajar terhadap pengajian ilmu balaghah: Satu tinjauan awal. Islamiyyat, 35(1), 93-101. https://ejournal.ukm.my/islamiyyat/article/view/4204.

Sulaiman, R. H. R. (2012). Penguasaan Balaghah Dalam Kalangan Pelajar IPT [Master dissertation, Universiti Malaya]. UM Students Repository

Wei, X., Saab, N., Admiraal, W. (2021). Assessment of cognitive, behavioral, and affective learning outcomes in massive open online courses: A systematic literature review. Computers \& Education 163. https://doi.org/10.1016/j.compedu.2020.104097.

Wei, X., Saab., N., Admiraal., W. (2021). Assessment of cognitive, behavioral, and affective learning outcomes in massive open online courses: A systematic literature review. Computers \& Education 163 (2021) 104097. http://www.elsevier.com/locate/compedu.

Yahya, H. (2016). Pembangunan prototaip aplikasi multimedia interaktif bahasa arab tahun satu [Unpublished master dissertation], Universiti Teknologi MARA (UiTM).

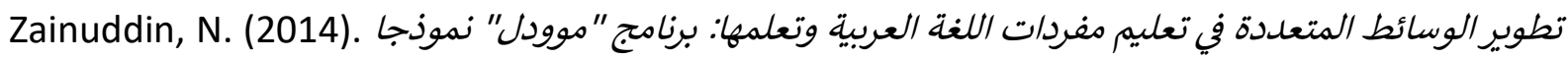
[Unpublished doctoral dissertation]. Universiti Islam Antarabangsa (UIAM). 
INTERNATIONAL JOURNAL OF ACADEMIC RESEARCH IN PROGRESSIVE EDUCATION AND DEVELOPMENT

Vol. 10, No. 3, 2021, E-ISSN: 2226-6348 @ 2021 HRMARS

Zaki, M., Hussin, M., \& Mezah, C. R. (2019). Pengantar balaghah Bahasa Melayu. Penerbit Universiti Putra Malaysia. 